\title{
Aprovechamiento recreativo de los hongos comestibles silvestres: casos de micoturismo en el mundo con énfasis en México
}

\author{
Recreational use of wild edible mushrooms: \\ mycological tourism in the world with an emphasis on Mexico
}

\author{
Andrea Jiménez-Ruiz a, Humberto Thomé-Ortiz ${ }^{\text {a* }}$, Angélica Espinoza-Ortega a , Ivonne Vizcarra Bordi a \\ *Autor de correspondencia: ${ }^{a}$ Universidad Autónoma del Estado de México, Instituto de Ciencias Agropecuarias y Rurales, \\ Instituto Literario Número 100 Oriente, Colonia Centro, Toluca Estado de México, México, CP 50000, \\ tel.: (0052) 7222965552, humbertothome@hotmail.com
}

\begin{abstract}
SUMMARY
In several countries around the world, including Mexico, there is a trend towards sustainable forest management, which includes non-timber forest resources and new non-extractive activities such as tourism. One of the resources that has arisen high interest in this regard are wild edible mushrooms, whose economic and cultural importance gives them the ability to be diversified in a wide range of products and services. Among the forms of diversification of mycological resources is tourism based on ild edible mushrooms, which is a recreational activity based on knowledge, identification, gathering and tasting of mushrooms. This literature review deals with the antecedents related to the recreational use of wild edible mushrooms and their challenges at a global level, emphasizing, in the case of Mexico, an analytical perspective of the processes of productive restructuring of the forest spaces. Mycological tourism is shown as an ambivalent activity, which is placed between a mercantilist vision on mycological resources and a forest management tool, based on investment processes, regulation and spatial planning. For this reason, it is necessary to analyze the productive transformations of the forests to know the contribution of mycological tourism in the social transformation and the improvement of the living conditions of rural communities.
\end{abstract}

Key words: mycological tourism, sustainable forest management, natural resources, cultural resources, rural development.

\section{RESUMEN}

En diversos países del mundo, incluido México, existe una tendencia hacia la gestión forestal sostenible, que contempla a los recursos forestales no maderables y nuevas actividades no extractivas como el turismo. Uno de los recursos que mayor interés ha despertado, en este sentido, son los hongos comestibles silvestres, cuya importancia económica y cultural les confiere la capacidad de ser diversificados en una amplia gama de productos y servicios. Dentro de las formas de diversificación de los recursos micológicos se encuentra el micoturismo, actividad recreativa basada en el conocimiento, identificación, recolección y degustación de los hongos. La presente revisión aborda los antecedentes relacionados con el aprovechamiento recreativo de los hongos comestibles silvestres y sus desafíos a nivel global, enfatizando en el caso de México desde una perspectiva analítica de los procesos de reestructuración productiva de los espacios forestales. El turismo micológico se muestra como una actividad ambivalente, que se debate entre una visión mercantilista sobre los recursos micológicos y una herramienta de manejo forestal, basada en procesos de inversión, regulación y ordenación del territorio. Por lo anterior, es necesario analizar las transformaciones productivas de los bosques para conocer la contribución del turismo micológico en la transformación social y la mejora de las condiciones de vida de las comunidades.

Palabras clave: turismo micológico, gestión forestal sustentable, recursos naturales, recursos culturales, desarrollo rural.

\section{INTRODUCCIÓN}

A lo largo de la historia, los bosques han constituido un elemento prioritario como medio de subsistencia humana debido a sus aportes a la diversidad ecosistémica y biológica, el aprovisionamiento de bienes y la prestación de diversos servicios ambientales (Carpentier et al. 2000). Dada la presión antrópica que enfrentan los bosques y su consecuente proceso de deforestación, los productos forestales no maderables han adquirido un papel relevante como recursos estratégicos para la gestión forestal sustentable (Boa 2004).

Actualmente, la gestión forestal enfrenta el reto de mitigar la pobreza y lograr la conservación del ambiente, mediante el aprovechamiento integral de los recursos y la diversificación de las actividades productivas (Salafsky y Wollenberg 2000). Dichas acciones orientadas a mejorar las condiciones de vida de las comunidades forestales, cuya población experimenta niveles altos de marginación (Secretaría del Convenio sobre la Diversidad Biológica 2009). 
Los impactos económicos negativos, producidos por el modelo económico neoliberal, han orillado a los territorios rurales a adoptar nuevas funciones como la conservación de la naturaleza, la producción local de calidad, las energías renovables y funciones culturales como el turismo.

Las actividades turísticas relacionadas con los bosques se basan en el interés social por un esparcimiento de calidad, ambientes saludables y escenarios naturales, donde se puedan experimentar sensaciones y emociones vinculadas con la ruralidad (Donaire y Gordi 2003). La globalización es el escenario donde acontece la turistización de los espacios rurales (Aguilar 2005), como resultado de las múltiples interacciones entre lo local y lo global, donde la búsqueda de identidad y el regreso a los orígenes son aspectos sustantivos en las formas de consumo en el capitalismo tardío (Lipovetsky y Serroy 2015).

De acuerdo con Lane (1994), en los productos del turismo rural se reflejan las características del lugar donde se desarrolla la actividad, una de sus motivaciones esenciales es el escape del estilo de la vida urbano, mediante la experimentación momentánea de la vida en el campo. Para ello se construye una oferta de ocio integrada que permita al viajero un contacto cercano con el entorno natural, al mismo tiempo que agrega valor a los recursos locales y puede ayudar a preservar el patrimonio rural (Thomé-Ortiz 2008). Lo anterior explica la proliferación de proyectos y productos turísticos en distintas zonas forestales alrededor del mundo que, mediante el principio de especialización territorial, atienda las demandas de un turismo cada vez más diferenciado (Sabaté et al. 2010).

Los hongos comestibles silvestres han sido percibidos como un recurso con capacidad de contribuir a la diversificación económica del medio rural, gracias a su potencial como recurso turístico (Lázaro 2008). En diferentes partes del mundo, han ganado un lugar protagónico como recursos centrales para la gestión integral de los espacios forestales (Martínez et al. 2010). De particular interés es la vinculación de los recursos micológicos con las actividades de ocio turístico, modalidad que ha sido denominada micoturismo y que se basa en la posibilidad de agregar valor a los hongos comestibles silvestres como un atractivo, natural y cultural, ligado al territorio.

Este trabajo tiene como objetivo presentar un panorama global del aprovechamiento recreativo de los hongos comestibles silvestres en México y el mundo, para analizar la restructuración productiva de los espacios forestales en el contexto de una economía global fuertemente orientada a los servicios. Para ello el texto se estructura de la siguiente manera: en el primer apartado se hace una revisión general del aprovechamiento recreativo de los hongos comestibles silvestres; posteriormente se describen las prácticas micoturísticas en el mundo; en tercer lugar se aborda el caso particular del micoturismo en México; el cuarto apartado explora si esta tipología turística puede ser una herramienta para la gestión forestal sostenible; finalmente se concluye que el aprovechamiento de los recursos mi- cológicos vinculados al turismo ilustra las nuevas tendencias de aprovechamiento forestal, enfocadas en productos forestales no maderables, entre las que se distinguen diferentes formas de aprovechamiento turístico, asociadas a factores sociales, económicos, políticos y etnológicos.

\section{EL APROVECHAMIENTO RECREATIVO DE LOS HONGOS COMESTIBLES SILVESTRES}

El turismo micológico o micoturismo, es una actividad recreativa en la que, a través de la observación, recolección y degustación, de los hongos comestibles silvestres sugiere un vínculo entre naturaleza y cultura, a través del concepto de patrimonio biocultural (Toledo y Barrera 2008). Es una actividad con contenido ambiental, que intenta transmitir a los turistas información sobre los recursos micológicos para su re-valoración (Lázaro 2008).

A partir del análisis de diversos casos de micoturismo en el mundo, se pueden concebir tres enfoques bajo los cuales se ha desarrollado la actividad: a) el enfoque micogastronómico, lo sitúa entre naturaleza y cultura, donde el turista se desplaza para degustar los recursos micológicos y al mismo tiempo hacer uso de los servicios e infraestructuras turísticas del entorno, en un tránsito que va de la tierra al plato (Lázaro 2008); b) el enfoque micopaisajístico en el cual la experiencia y el disfrute están ligados al medio natural, a la apreciación de paisajes y la observación de otros elementos naturales alrededor de los hongos (Martínez et al. 2010) y; c) el enfoque etnomicológico, relacionado con el conocimiento tradicional de los grupos étnicos, incluidos los usos y significados (Ruán-Soto et al. 2004, Toledo y Barrera 2008), que permitan asegurar su reproducción social a través del tiempo y el espacio. En algunas regiones sin conocimiento tradicional micológico, el micoturismo se puede concebir a partir de los dos primeros enfoques, sin embargo, en países como México los hongos siempre están ligados a los recolectores tradicionales y su cultura, por lo que la actividad recreativa suele estar más relacionada con el tercer enfoque.

En este sentido Jiménez-Ruiz et al. (2016) definen al micoturismo como una "modalidad híbrida del ocio turístico que se construye a partir de una oferta cultural, étnica, ecológica y gastronómica, situadas en un punto intermedio entre la naturaleza y la cultura".

Cada enfoque determina las actividades micoturísticas que se puedan ofertar y ninguno de ellos es excluyente del otro. Uno de los retos principales para la implementación del micoturismo, es crear vínculos entre los distintos elementos del territorio y generar un aprovechamiento pleno de los recursos locales, que mejore la calidad de vida e incentive la protección de los recursos micológicos.

De acuerdo con lo anterior, el desarrollo del micoturismo depende de un conjunto de variables, que permiten o no su implementación, en su mayoría ligadas a la configuración natural, etnológica, económica, cultural y política de cada territorio. 
Micoturismo: la dimensión recreativa de los hongos comestibles silvestres. El micoturismo engloba dentro de sus variantes prácticas activas y otras de carácter contemplativo. Las principales acciones son la recolección recreativa, el consumo gastronómico, la educación ambiental y el comercio de productos alimentarios (Martínez 2010), además de la interacción entre culturas a partir del conocimiento tradicional sobre los hongos comestibles silvestres (Jiménez-Ruiz et al. 2016).

Siguiendo a Lázaro (2008), dichas actividades podrían clasificarse en dos grupos: a) productos turísticos con precio, como rutas micológicas guiadas; paquetes micoturísticos que comprenden un fin de semana, talleres de identificación de especies combinados con otros recursos territoriales tanto naturales, como culturales o paisajísticos y jornadas gastronómicas $\mathrm{y}, \mathrm{b}$ ) productos micológicos sin precio, como rutas auto guiadas. Ello nos permite apreciar una de las principales ambivalencias de la gestión turística de los hongos comestibles silvestres, es la divergencia entre su carácter como bien común de libre acceso para habitantes de las comunidades forestales y su carácter como un recurso con valor de mercado de cara al turismo. En estos términos el despliegue del capital rural (natural y cultural) con que cuente cada territorio es un factor determinante en su capacidad de atraer flujos turísticos (Garrod et al. 2006). En todas las actividades antes mencionadas subyacen formas de capital rural, cuya tendencia es transitar de las funciones convencionales de regulación y aprovisionamiento, a su aprovechamiento como servicios culturales.

De acuerdo con Bennett et al. (2012), el turismo rural depende de siete formas de capital: natural, que integra la reserva de recursos naturales disponibles; cultural, que incluye prácticas, tradiciones, etnoconocimiento e identidad local; físico, que engloba la infraestructura para el desarrollo del turismo; social, que considera las redes sociales y la organización alrededor del turismo; financiero, que incorpora los recursos económicos necesarios para la implementación del turismo; humano, que considera conocimientos, habilidades y atributos que poseen los actores locales, y político, que incluye relaciones con instituciones, regulaciones e influencia de los programas que facilitan la transformación los activos del capital rural en recursos turísticos.

El estado del arte del micoturismo. El estudio del aprovechamiento de los hongos comestibles silvestres es relativamente reciente (Boa 2004). Las principales investigaciones en la materia han versado sobre aspectos biológicos (Montoya et al. 2014), metodológicos (Martínez et al. 2011), económicos (Fernández et al. 2012), y sociales (Burrola et al. 2012). La etnomicología ha sido un referente importante para conocer lo relativo a su aprovechamiento y usos culturales (Moreno et al. 2001), sin embargo, el estudio de su faceta recreativa es incipiente.

En Europa se ha conformado la Red de Parques Micológicos, integrada por montes productivos y regulados, donde se conducen investigaciones enfocadas en la sus- tentabilidad, la diversificación productiva y el aprovechamiento recreativo de los hongos comestibles silvestres. La Red está integrada por ocho parques, cuatro de ellos ubicados en España, dos en Francia, uno en Portugal y otro en Canadá. La creación de estos parques, es el punto de partida para el denominado Observatorio Europeo de Micología que tiene como finalidad recabar datos homogéneos y generar indicadores sobre producción, recolección, comercialización, micoturismo y sostenibilidad en las regiones participantes (Micosylva 2013).

La información e investigaciones sobre el aprovechamiento recreativo de los hongos comestibles silvestres surgen desde distintas aristas. Entre las principales investigaciones, con enfoque sociológico, se encuentra el trabajo descriptivo de Lázaro (2008), en el que se realiza un balance general del micoturismo en España, mediante el análisis de la capacidad de diversificación productiva de estos hongos y algunas pautas para la gestión sostenible de la actividad, que incluye aspectos sociales, económicos y ambientales. El trabajo de Thomé-Ortiz (2016) indaga el papel del aprovechamiento recreativo de los hongos comestibles silvestres como estrategia de desarrollo rural en el centro de México, mediante el estudio del conocimiento, uso y las prácticas sociales alrededor de ellos, insumos fundamentales para el desarrollo del micoturismo.

Desde la perspectiva económica, el estudio de Martínez-Peña et al. (2011) se enfoca en el potencial económico de los recursos micológicos desde las vertientes ecológica y turística. Otros estudios (De Frutos et al. 2011) han analizado el potencial de generación de empleo e ingresos que los proyectos micoturísticos pueden aportar.

Martínez-Peña et al. (2003) propone una metodología para regular la recolección de distintas especies de hongos comestibles silvestres dentro del marco de ordenación de los recursos forestales no maderables en España. En otro estudio, Martínez (2010) se enfoca en el caso de la provincia de Soria, a partir del modelo gestión micológica MYAS (Micología y Aprovechamiento Sostenible), donde analiza la influencia de la actividad micoturística en la promoción del desarrollo local.

De Castro (2009) desarrolla un estudio, desde la perspectiva ecológica, resaltando el valor nacional que los recursos micológicos tienen en Portugal, apoyados en los enfoques cartográfico, biológico y ecológico. Discute sobre el establecimiento de normas para la gestión y el uso integrado de los hongos comestibles silvestres basado en acciones de educación ambiental y agregación de valor. Por su parte, Thomé-Ortiz (2016) aborda el potencial turístico de estos hongos en una comunidad indígena del centro de México, bajo un análisis cualitativo de los recursos micológicos y sus sistemas socioecológicos asociados. La Asociación Micológica Chilena realiza investigaciones sobre la conservación de los hongos comestibles silvestres y acercamientos al marco legal para su recolección. Además, se han esforzado en la difusión y conservación de distintas especies del Patrimonio Fúngico de Chile (Furci y Repetto 2012). 
El trabajo de Knigth (2014) aborda el caso de la provincia de Kalloni en Macedonia, lugar que en temporada de hongos genera un retorno de sus antiguos habitantes para recolectar hongos, después del éxodo sufrido en los años cuarenta, tras la guerra civil. Este trabajo analiza la dimensión recreativa de los hongos comestibles silvestres desde una perspectiva antropológica en donde la nostalgia, la identidad, la memoria alimentaria y el territorio juegan un papel importante en la construcción social de la identidad histórica de este pueblo.

La investigación de Thomé-Ortiz (2015) versa sobre los escenarios del aprovechamiento recreativo de los hongos comestibles silvestres en México, desarrolla un enfoque social y multidisciplinario que explora las posibilidades de un modelo de gestión turística de los recursos micológicos.

La mayoría de las investigaciones sobre la actividad micoturística abordan estudios de caso sobre las comunidades donde se ha implementado, con énfasis en sus impactos económicos, ecológicos y sociales. Por otra parte, existen importantes áreas de oportunidad para la investigación de este recurso como el análisis antropológico que permita indagar en su valor simbólico y que integra la variable de etnicidad, presente en muchas comunidades recolectoras. Es necesario abonar a estudios con enfoque de género, como el realizado por Garibay-Orijel et al. (2012), en los que se analiza el papel de las mujeres en la recolección, procesamiento y comercialización de los hongos comestibles silvestres como parte de su identidad cultural, vinculados al aprovechamiento turístico. Así como trabajos que aborden los aspectos jurídicos, regulatorios y normativos alrededor del aprovechamiento de dichos recursos, que contribuyan a la generación de un sistema de gestión micológico para su aprovechamiento, sin poner en riesgo su diversidad y disponibilidad (McLain 2008). No se reportan trabajos que hagan un estudio sistemático de la demanda de micoturistas y del perfil de los visitantes que desarrollan estas prácticas.

Pese a la proliferación de iniciativas turísticas para el aprovechamiento de los hongos comestibles silvestres, aún es escasa la producción científica respecto a este fenómeno (Benítez et al. 2013). A la par, la falta de gestión, vinculación e información, dificulta el correcto desarrollo del micoturismo, lo que pone en relieve la pertinencia de generar conocimiento científico básico acerca de los beneficios y riesgos que entraña esta actividad en ecosistemas altamente vulnerables.

\section{LAS PRÁCTICAS MICOTURÍSTICAS EN EL MUNDO}

La emergencia del micoturismo en el mundo se atribuye a la convergencia de diferentes factores como: i) la actuación del Estado a través de políticas públicas para la búsqueda de alternativas de gestión forestal (Lázaro 2008); ii) el auge de productos alimentarios de calidad, asociados con territorios específicos y vinculados con la sociedad a través del turismo (Mason 2009); y iii) la creciente necesidad de la sociedad urbana por estar en contacto con la naturaleza (Aguilar 2005). Además de estas tendencias globales es evidente que cada territorio presenta particularidades naturales, culturales y etnológicas, que inciden en la inquietud de las propias comunidades por fomentar el aprovechamiento recreativo de los hongos comestibles silvestres. A continuación se presentan algunos de los casos más representativos del micoturismo en el mundo.

España. Es el país pionero en el desarrollo de micoturismo a nivel mundial. Ello debido a que la gestión de los hongos comestibles silvestres forma parte de las políticas de desarrollo rural de la Unión Europea y cuenta con recursos financieros de la Iniciativa Comunitaria LEADER y el programa LIFE MYAS, que para el periodo 2009 - 2011, le fue asignado un presupuesto de $€ 1.798 .452,26$ (MartínezPeña et al. 2011), para el aprovechamiento y conservación de los recursos micológicos incluyendo la cuestión turística. El modelo español tiene como eje central la regulación ordenada de los hongos (Lázaro 2008), para con ello generar beneficios económicos, la preservación de la cultura micológica y la protección y reproducción de los hongos a largo plazo (Egli et al. 2006).

Es claro que el modelo español tiene una orientación hacia el mercado, basada en incentivos para la creación de empresas de transformación de hongos, modelos micoturísticos comerciales, restaurantes micogastronómicos y el establecimiento de lonjas micológicas, entre sus principales actividades. Un ejemplo de ello es el proyecto LEADER "Micología y Calidad" que agrupa más de cien establecimientos micológicos, configurando una oferta micoturística única a nivel global (Micosylva 2013).

Además de la visión de negocios que subyace a este modelo, las acciones estratégicas contemplan un sistema de información micológica (SIM) denominado Micodata, el cual tiene como tarea aportar datos dinámicos para la localización y estimación de los recursos micológicos (Micodata 2013).

España representa el único modelo integral de recursos micológicos que puede resumirse en cuatro características esenciales: i) Un soporte financiero institucional basado en el apoyo del Estado a través de diversos programas; ii) Un marco regulatorio que incide en una normatividad clara sobre el aprovechamiento y gestión de los hongos comestibles silvestres; iii) Un Sistema de Información Micológica que aporta datos dinámicos sobre el recurso micológico y; iv) La existencia de un mercado nutrido por las nuevas tendencias del consumo turístico y alimentario.

Portugal. De igual manera que en España, el micoturismo se ha desarrollado a partir de estudios y aproximaciones técnicas basadas en el conocimiento sobre los recursos micológicos, su ubicación y su dimensión ecológica. Se trata de una iniciativa con una fuerte orientación al mercado, cuyo impulso tiene una estrecha relación con la iniciativa privada. 
En el modelo micoturístico portugués destacan los enfoques micogastronómico y micopaisajístico, aspectos que influyen en el desarrollo de una oferta de turismo alimentario y de naturaleza. No existen programas ni apoyos del Estado, aspecto que ha limitado el repunte de este sector. Lo anterior, ilustra las limitaciones que enfrentan las iniciativas privadas y los esfuerzos particulares para la gestión turística de hongos comestibles silvestres, a diferencia de aquellos con iniciativas estratégicas inscritas en programas de más amplio calado (De Castro 2009).

Francia. Este país se ha integrado a la Red Micosylva de Parques Micológicos a través del registro de los parques de la Dordogne y de Midi Pyréneés. Para ello, se ha seguido el modelo de laboratorios micológicos experimentales que intentan ser unidades de gestión para el desarrollo territorial, a través del manejo adecuado y la justa valorización de los recursos micológicos. Una característica esencial del modelo francés es que dentro de su propuesta micoturística se concibe la importancia de la educación ambiental como un componente de las actividades recreativas.

Este caso ilustra la importancia de las acciones de colaboración para el desarrollo de las propuestas micoturísticas. En este sentido, ha sido crucial la creación y fortaleci- miento entre los diferentes actores involucrados entre los que destacan: las instituciones públicas, la academia y los pequeños productores locales, lo cual ha desembocado en una oferta micoturística centrada en la conservación y con un alcance regional (Micosylva 2013).

Escocia. Los primeros intentos del micoturismo en Escocia surgieron hace una década, a partir del interés académico sobre las particularidades ecológicas del territorio, situación que determinó su idoneidad para conocer la diversidad fúngica de sus bosques. Este modelo se enfoca hacia el turismo social, la educación ambiental y la preservación de los bosques. Sus primeras iniciativas han tenido un enfoque micopaisajístico y se han desarrollado en la zona norte de Perthshire.

La actividad micoturística desarrollada, tiene un fuerte componente científico y ha sido apoyada por la Sociedad Danesa de Micología, aspecto que aporta soporte científico a las prácticas turísticas que son guiadas por micólogos. Comparado con los casos de España y Francia, no existe un modelo micoturístico claramente desarrollado, tampoco se presenta una participación del Estado a través de políticas de micosilvicultura, pero sí es evidente la importancia de la academia en el impulso de la actividad (Scottish Fungi 2014).

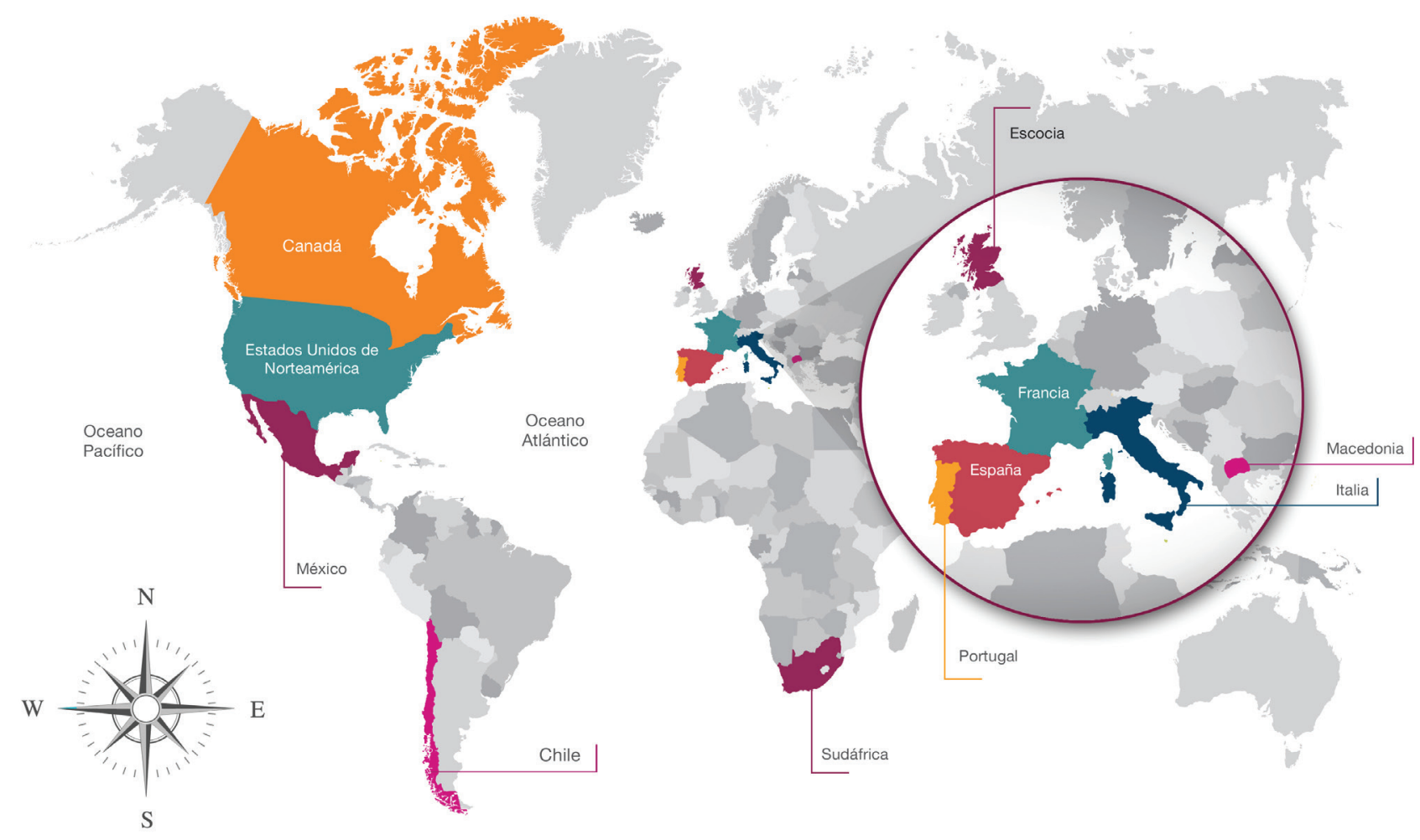

Fuente: elaboración propia con base en la investigación.

Figura 1. Sitios con práctica micoturística en el mundo.

Sites where mycotourism takes place in the world. 
Italia. En este país juega un papel fundamental el asociativismo alrededor de los hongos. Existen asociaciones, aficionadas y especializadas, cuyo objetivo es generar conocimiento sobre los hongos comestibles silvestres del mediterráneo. Los trabajos de estos grupos se enfocan en las áreas de cartografía, taxonomía, nomenclatura, ecología y legislación (Zambonelli et al. 2015, Simone et al. 2017).

$\mathrm{Al}$ igual que en el caso francés, se ha manifestado el interés por integrar algunos de estos bosques a la Red de Parques Micológicos del Instituto Europeo de Micología (Micosylva 2013). Ciertamente, en estas iniciativas subyace el enfoque micopaisajístico y micogastronómico, con lo que se privilegian los atributos naturales y culturales, vinculados con los hongos comestibles silvestres. Sin embargo, es necesario crear marcos apropiados para la regulación y ordenamiento de la actividad micoturística.

Sudáfrica. La Academia Sudafricana de Hongos Gourmet es una asociación privada, encargada de capacitar a proveedores de recursos micológicos, desde un enfoque biotecnológico, empresarial y económico. Ofrece servicios basados en los hongos comestibles silvestres como eventos gastronómicos y programas de educación a distancia, con el apoyo financiero del Fondo Mundial para la Naturaleza de Sudáfrica. En cuanto a la práctica micoturística, se realizan actividades de recolección, difusión de la cultura micológica y eventos micogastronómicos. Sin embrago, estos eventos son limitados por la baja disponibilidad de hongos comestibles silvestres, siendo substituidos en ocasiones por hongos cultivados, dejando fuera el vínculo entre el turista y el territorio lo que cuestiona ampliamente el desarrollo de una verdadera experiencia micoturística (Le mycotourisme 2014).

Canadá. La riqueza micológica de este país y el interés de un grupo de especialistas son los elementos que explican la emergencia del micoturismo en Canadá. Se han documentado diferentes iniciativas que incluyen la transformación agroindustrial de hongos comestibles silvestres, emprendimientos turísticos y propuestas de educación ambiental. El micoturismo tiene una orientación económica, que busca incidir favorablemente en las economías locales, por lo que se le concibe como una política de desarrollo (Mycosylva 2013).

Estados Unidos. Este país cuenta con distintas asociaciones micológicas encargadas de difundir la cultura micológica y su disfrute gastronómico; así como la importancia ecológica y el valor cultural de los hongos. Por parte de la academia se han generado además investigaciones enfocadas en el estudio de los bosques y su gestión a partir de la categorización, monitoreo, seguimiento y vigilancia del aprovechamiento de los hongos comestibles silvestres (McLain 2008).

Como en la mayoría de los casos la gestión de los recursos micológicos, parte de un conjunto de actividades dispersas como ferias, recorridos para recolección y una oferta culinaria incipiente. La academia impulsa actividades que han sido de vital importancia, particularmente en lo que respecta al conocimiento de los recursos micológicos en la zona (Vantomme 2003, Boa 2004).

Macedonia. La temporada de hongos en el territorio de Kalloni despliega un marcador de identidad territorial, asociado con los conocimientos tradicionales ecológicos sobre los hongos comestibles silvestres, la relación con la naturaleza y la cultura local. De acuerdo con ello, el micoturismo en Macedonia representa una forma de turismo de nostalgia, a través de la cual los visitantes provenientes de las ciudades periféricas se reconectan con los orígenes y se genera una derrama económica, no se trata de un producto turístico orientado al mercado sino de una práctica colectiva presente en la memoria cultural de sus practicantes (Knight 2014).

Este caso es de particular importancia, porque revela que la dimensión recreatia de los hongos comestibles silvestres no está limitada a su dimensión de mercado, sino que tiene un significado lúdico de alto valor social para aquellos que cotidianamente recolectan y consumen hongos.

\section{MICOTURISMO EN MÉXICO}

La emergencia del micoturismo en México responde a un conjunto de situaciones críticas que afectan a las comunidades forestales (Thomé-Ortiz 2016), en su mayoría pertenecientes a grupos étnicos altamente marginados. Esta actividad se ha desarrollado a partir de diferentes perspectivas: los destinos micoturísticos, las ferias alimentarias, las exposiciones y los eventos académicos especializados en hongos (Pérez-Silva y Herrera 2015). El caso de México ilustra un ejemplo significativo debido a los avances en el conocimiento ecológico y etnomicológico. Igualmente sobresale la riqueza cultural de los mercados y tianguis de las localidades aledañas a los bosques (Moreno-Fuentes et al. 2001, Garibay-Orijel et al. 2007, Ruán-Soto et al. 2009. Burrola-Aguilar et al. 2012). Dichos antecedentes son un indicativo de la importancia que tiene el aprovechamiento integral de los recursos micológicos en México.

Los destinos micoturísticos. A pesar de que el uso de los hongos comestibles silvestres y alucinógenos data de la época prehispánica, los estudios micológicos al respecto, surgen sobre todo en la década de los cincuenta en la población de Huautla de Jiménez en Oaxaca, donde una pequeña comunidad indígena usaba los hongos sagrados (alucinógenos), con fines religiosos, morales o de salud. Derivado de ello tomaron lugar desplazamientos de personas interesadas en experimentar su consumo (Guzmán 2016). Sin embargo, estas prácticas se enfrentan a diversos debates éticos y jurídicos que las distancian de la concepción contemporánea del ocio recreativo. 
Yoricostio Michoacán fue uno de los primeros lugares en desarrollar una práctica micoturística en México. La iniciativa se gestó con el apoyo de la Universidad Michoacana de San Nicolás de Hidalgo y el Ayuntamiento de Morelia (Gómez y Zamora 2012). Aunado a ello, la Asociación de Recolectores de Hongos denominada La Villita, se apropiaron del proyecto mediante la oferta de recorridos micoturísticos, basados en la recolección recreativa, la educación ambiental y la degustación de la cocina tradicional.

Otra oferta se refiere a la iniciativa "Micoturismo México", la cual surgió de algunos estudios científicos sobre la micodiversidad del municipio de Tequila, en el Estado de Jalisco. Dichos datos fueron el punto de partida para estructurar una oferta micoturística basada en el conocimiento científico de los hongos, el senderismo interpretativo y la degustación de la cocina tradicional (Villaseñor et al. 2011, Padilla 2014). A diferencia del caso de Michoacán, en Tequila, el proyecto es operado por el sector académico y una pequeña empresa especializada en turismo alternativo.

Otros sitios en los que se llevan a cabo recorridos micoturísticos son Tlaxcala en colaboración con la Universidad Autónoma de Tlaxcala, además de Amanalco de Becerra y Cacalomacán en el estado de México, donde actualmente se llevan a cabo estudios para su implementación (Zizumbo-Villareal et al. 2012, De la Garza 2017).

Las ferias del hongo. Las ferias alimentarias son plataformas de exhibición para los productos locales, cuya finalidad es mejorar la economía, a través de la comercialización de alimentos producidos localmente. En diferentes lugares de México ha surgido el interés por realizar ferias temáticas alrededor de los hongos comestibles silvestres. En estas ferias se comercializan los hongos del territorio, se ofrecen productos procesados, gastronomía, artesanías y servicios turísticos.

Las ferias alimentarias son un medio propicio para promover el micoturismo; debido a que sirven como eventos para la identificación del territorio y permiten acercarse a la cultura local de un modo más vivencial (Armesto y Gómez 2004).

En México existen dos ferias de hongos comestibles silvestres con gran tradición: la de Cuajimoloyas, Oaxaca, y la de Senguio, Michoacán, debido a su trayectoria de más de 15 años, presentan una oferta estructurada y una sólida plataforma de promoción. A lo largo del país, se generan anualmente eventos académicos referentes a los hongos comestibles silvestres, como los organizados por la Sociedad Mexicana de Micología, que datan de 1969 (Pérez-Silva y Herrera 2015), otros por el Grupo Interdisciplinario para el Desarrollo de la Etnomicología en México (GIDEM 2014) y diversas Universidades, en los que esporádicamente se organizan recorridos micoturisticos. Se observa una constante proliferación de ferias, iniciativas que parten de grupos heterogéneos. Estos eventos son concretados en un conglomerado de esfuerzos dispersos que, aunado con la falta de una política de Estado referente a la micosilvicultura, su ordenamiento y regulación, inciden en una inadecuada gestión de los recursos micológicos.

\section{A MANERA DE CONCLUSIÓN: ¿ES EL MICOTURISMO UNA HERRAMIENTA PARA LA GESTIÓN FORESTAL SOSTENIBLE?}

Convencionalmente la gestión forestal ha privilegiado un enfoque monofuncional y extractivo, sesgado a los recursos maderables, lo que ha soslayado el conjunto de servicios que los bosques brindan a la sociedad. En las últimas décadas, las políticas de algunos países se han centrado en estas dimensiones de los bosques que habían permanecido ocultas, y que tienden a una gestión forestal multifuncional (Alvarado y Benítez 2009).

Actualmente, el reto del aprovechamiento de los bosques, es desarrollar una gestión forestal sostenible para la satisfacción equitativa de las necesidades humanas presentes y futuras, desde un triple enfoque: económico, ambiental y sociocultural. La tendencia es que los bosques se conviertan en espacios multifuncionales para el logro de la sustentabilidad forestal (Boyd y Banzhaf 2007).

El enfoque económico plantea la generación de empleos e ingresos para las economías locales, mediante el desarrollo de infraestructura, el aprovechamiento de productos forestales no maderables y la comercialización de servicios ambientales y culturales del bosque, entre los que se encuentra el turismo. El enfoque sociocultural plantea un aumento de la participación social en las iniciativas de gestión del bosque y un acceso equitativo a los recursos locales, para lo que es necesario el aprendizaje colectivo, la cooperación y confianza entre los actores. El enfoque ambiental persigue mantener los recursos genéticos con inclusión de especies vulnerables, amenazadas y en peligro; así como la diversidad de ecosistemas (FAO 2014).

En el caso particular de los hongos comestibles silvestres, se adaptan de manera apropiada a las tendencias multifuncionales de los espacios forestales. Además cumplen importantes funciones para la preservación del ecosistema, su participación en el ciclo orgánico permite que otras especies se consoliden y sobrevivan, son agentes reguladores del equilibrio ecológico que contribuyen al reciclaje de nutrientes mediante la descomposición de residuos que benefician la fertilidad del suelo, contribuyendo así a la salud y preservación de todo el sistema forestal (Montoya y Orrego 2012).

Los hongos comestibles silvestres son recursos con importancia económica derivada de la venta del producto para la subsistencia de los recolectores (Garibay-Orijel et al. 2009), su transformación en productos agroindustriales (Alvarado et al. 2015) y su apropiación turística. Además, cumplen con importantes funciones socioculturales debido a sus atributos estéticos materializados en el paisaje, la gastronomía y la cultura (Arana et al. 2014). Su aprovechamiento estimula la organización social y la conservación de los conocimientos tradicionales referentes a su recolección y uso (Alvarado y Benítez 2009). 
Desde esta perspectiva es importante cuestionarse el papel del micoturismo como instrumento de planificación y ejecución de acciones para la gestión forestal sostenible, al mismo tiempo que refleje una aceptación de las comunidades y una adaptación a las transformaciones globales de los espacios rurales (Micosylva 2013). El papel del micoturismo respecto a la gestión forestal sostenible se plantea ambivalente, por una parte es una forma de aprovechamiento multifuncional del territorio, por la otra, refleja una actividad que requiere una alta especialización productiva, sirviendo como parteaguas de inclusión o exclusión a la comunidad, preservar o depredar los recursos micológicos según sea el modelo de turismo micológico subyacente.

Ciertamente, el aprovechamiento de los hongos comestibles silvestres ya sea a través de su comercialización en fresco, en productos transformados o desde el micoturismo, repercute de manera positiva en el desarrollo económico de los espacios rurales, pero puede generar problemas sociales y de sostenibilidad del recurso (Martínez 2010). La implementación de prácticas insostenibles de recolección repercute negativamente en la conservación del recurso. La inadecuada ejecución del micoturismo puede generar impactos negativos como la contaminación, el desequilibrio ambiental o la destrucción del mismo recurso.

Como se ha podido observar el aprovechamiento micológico, ya sea en su faceta recreativa o comercial, carece de un marco regulatorio y una política de ordenamiento en la mayoría de los casos. El modelo español es un referente importante, donde se cuenta con leyes de protección y regulación, basadas en conocimientos científicos básicos que permiten detectar las áreas de recolección, las especies y cantidades factibles para recolección. Para ello se desarrolla un sistema de permisos con diferentes categorías, que permite generar ingresos públicos y disminuir la presión sobre el recurso.

Indudablemente, es una propuesta interesante que a partir de la inversión pública ha desembocado en el crecimiento del sector micológico en el norte de España, sin embargo, su abierta orientación hacia el mercado no necesariamente corresponde con la realidad latinoamericana donde la recolección de hongos se asocia con grupos étnicos y silvicultura de subsistencia.

La revaloración económica de los hongos comestibles silvestres asociada al turismo tiene como fundamento una estrecha relación con la investigación científica. Un ejemplo de ello son los avances sobre la domesticación de algunas especies (Reyna y García 2014, Alvarado et al. 2015), lo cual puede ser una herramienta para favorecer el equilibrio entre conservación y desarrollo.

Uno de los aspectos críticos del micoturismo es que se trata de una actividad que intenta conciliar los objetivos de protección y crecimiento que muchas veces se extrapolan en las lógicas de conservación y desarrollo.

En los diferentes casos analizados se observa como el micoturismo puede ser un factor positivo para la gestión forestal sostenible. Al implementar aspectos del pilar eco- nómico, como la generación de empleos y comercialización de productos forestales no maderables; a través de prácticas adecuadas de recolección, realización de eventos académicos y de educación ambiental se contribuye a la conservación del recurso micológico en el pilar medioambiental; los vínculos y participación generados entre los actores que intervienen en la planeación y desarrollo de la actividad inciden en el nivel sociocultural que dicho modelo de gestión plantea.

La acumulación de diversos capitales rurales ha sido un elemento clave en el desarrollo de experiencias micoturísticas exitosas. Se percibe que los modelos mejor instrumentados, despliegan siete formas de capital (Benet et al. 2012): i) natural (disponibilidad micológica), ii) cultural (conocimientos sobre los hongos comestibles silvestres), iii) físico (servicio de hospedaje, restaurantes micológicos), iv) social (comités de turismo), v) financiero (fondeadoras y programas), vi) humano (personal capacitado y especializado), y vii) político (normatividad, regulación proyectos nacionales).

Los modelos de micoturismo que se han implementado alrededor del mundo son muy diversos, desafortunadamente, la mayoría de las iniciativas micoturísticas contienen un fuerte sesgo hacia el capital natural, no confiriendo importancia a los otros distintos capitales necesarios para el desarrollo de un producto turístico integral.

En Europa, los modelos han sido desplegados desde las iniciativas gubernamentales y la integración de distintos elementos del capital rural, mediante la intermediación del sector académico y la participación de diferentes tipos de asociaciones interesadas en la recolección.

Es necesario fomentar el micoturismo basado en un modelo integral que incorpore sistemas de información micológica, basados en estudios etnomicológicos y en el conocimiento tradicional de las comunidades rurales; además de mecanismos de regulación referentes a la producción, recolección, comercialización de los hongos comestibles silvestres. Una característica muy importante para el desarrollo de la actividad micoturística es la integración de las comunidades forestales y su capital rural (natural, cultural, físico, social, político, financiero y humano) participante, lo cual contribuirá, en mayor medida, a las transformaciones económicas, ambientales y socioculturales, que plantea el modelo de gestión forestal sustentable.

\section{AGRADECIMIENTOS}

Agradecemos el apoyo de los proyectos de investigación "Los hongos comestibles silvestres y sus escenarios turísticos. Laboratorio Social de Micoturismo", financiado por el programa de Investigación Científica, Innovación y Desarrollo UAEM 2014, y del proyecto de investigación: "Evaluación de la dimensión recreativa de los hongos comestibles silvestres, su interés socioeconómico y sus perspectivas de desarrollo rural", financiado por CONACYT - SEP Ciencia Básica 2014. 


\section{REFERENCIAS}

Aguilar E. 2005. Patrimonio y globalización: el recurso de la cultura en las Políticas de Desarrollo Europeas. Cuadernos de Antropología Social 21: 51-69.

Alvarado G, G Benítez. 2009. El enfoque de agroecosistemas como una forma de intervención científica en la recolección de hongos silvestres comestibles. Tropical and Subtropical Agroecosystems 10: 531-539.

Alvarado G, G Mata, G Benítez. 2015. Importancia de la domesticación en la conservación de los hongos silvestres comestibles en México. Bosque 36(2): 151-161. DOI: 10.4067/ S0717-92002015000200001.

Arana Y, C Burrola, R Origel, y S Franco. 2014. Obtención de cepas y producción de inóculo de cinco especies de hongos silvestres comestibles de alta montaña en el centro de México. Revista Chapingo, Serie Ciencias Forestales y del Ambiente 20(3): 213-226. DOI: 10.5154/r.rchscfa.2014.04.017.

Armesto X, B Gómez. 2004. Productos alimentarios de calidad, turismo y desarrollo local. Cuadernos Geográficos 84(1): 83-94.

Benítez G, G Alvarado, M Nava, A Pérez. 2013. Análisis del marco regulatorio en el aprovechamiento de los hongos silvestres comestibles en México. Revista Chapingo Serie Ciencias Forestales y del Ambiente 19(3): 363-374. DOI: 10.5154/r.rchscfa.2012.09.055.

Bennett N, R Lemelin, R Koster, I Budke. 2012. A capital assets framework for appraising and building capacity for tourism development in aboriginal protected area gateway communities. Tourism Management 33(4): 752-766. DOI: 10.1016/j.tourman.2011.08.009.

Boa E. 2004. Non-wood forest products. Wild Edible Fungi. A Global Overview of Their Use and Importance to People. FAO. 159 p.

Boyd J, S Banzhaf. 2007. What are ecosystem services? The need for standardized environmental accounting units. Ecological Economics 63(2-3): 616-626. DOI: 10.1016/j.ecolecon.2007.01.002.

Burrola C, O Montiel, R Garibay, L Zizumbo. 2012. Conocimiento tradicional y aprovechamiento de los hongos comestibles silvestres en la región de Amanalco, Estado de México. Revista Mexicana de Micología 35: 1-16.

Carpentier CL, S Vosti, J Witcover. 2000. Intensified Production Systems on Western Brazilian Amazon Settlement Farms: Could They Save the Forest? Agriculture, Ecosystems and Environment 82(1-3): 73-88. DOI: 10.1016/S01678809(00)00217-6.

De Castro S. 2009. Micoturismo: enquadramento estratégico em áreas protegidas. Tesis Doctoral. Portugal. Universidad de Técnica de Lisboa. $81 \mathrm{p}$.

De Frutos P, F Martínez, S Esteban. 2011. El turismo micológico como fuente de ingresos y empleo en el medio rural. El caso de Castilla y León. Estudios de Economía Aplicada 29(1): 279-307.

De La Garza PM. 2017. Integración del Patrimonio Biocultural sobre los hongos como estrategia de desarrollo sostenible en el Parque ecoturístico de Cacalomacán, estado de México. México DF, México. Tesis de Maestría en Ciencias Agropecuarias y Recursos Naturales. Instituto de Ciencias Agropecuarias y Rurales de la Universidad Autónoma del Estado de México. 75 p.
Donaire J, J Gordi. 2003. Bosque y Turismo. Boletín de la A.G.E. 35: 207-221.

Egli S, M Peter, C Buser, W Stahel, F Ayer. 2006. Mushroom Picking Does Not Impair Future Harvests. Results of a Long Term Study in Switzerland. Biological Conservation 129 (2): 271-276. DOI: 10.1016/j.biocon.2005.10.042.

Fernández M, C Barroetaveña, V Bassani, F Ríos. 2012. Rentabilidad del aprovechamiento del hongo comestible Suillus luteus para productores forestales y para familias rurales de la zona cordillerana de la provincia del Chubut, Argentina. Bosque 33(1): 43-52. DOI: $10.4067 / \mathrm{S} 0717-$ 92002012000100005.

Food and Agriculture Organization of the United Nations (FAO). 2014. Gestión Forestal Sostenible. Consultado 10 ene. 2016. Disponible en http://www.fao.org/forestry/sfm/es .

Furci GN, F Repetto-Giavelli. 2012. Catálogo preliminar de los hongos del valle La Paciencia, sur-este de Tierra del Fuego, Chile. Anales del Instituto de la Patagonia 40(2): 4754. DOI: 10.4067/S0718-686X2012000200004.

Garibay-Orijel R, J Caballero, A Estrada-Torres, J Cifuentes. 2007. Understanding cultural significance, The edible mushrooms case. Journal of Ethnobiology and Ethnomedicine 3: (4) DOI: 10.1186/1746-4269-3-4.

Garibay-Orijel R, J Córdova, J Cifuentes, R Valenzuela, A Estrada-Torres, A Kong. 2009. Integrating wild mushrooms use into a model of sustainable management for indigenous community forests. Forest Ecology and Management 258(2): 122-131. DOI: 10.1016/j.foreco.2009.03.051.

Garibay-Orijel RG, A Ramírez-Terrazo, M Ordaz-Velázquez. 2012. Women care about local knowledge, experiences from ethnomycology. Journal of Ethnobiology and Ethnomedicine 8(25): 1-13. DOI: 10.1186/1746-4269-8-25.

Garrod B, R Wornell, R Youell. 2006. Re-conceptualising rural resources as countryside capital: The case of rural tourism. Journal of Rural Studies 22(1): 117-128. DOI: 10.1016/j.jrurstud.2005.08.001.

Gómez P, E Zamora. 2012. Los hongos silvestres comestibles en Yoricostio, México. In Sánchez J, B Mata eds. Hongos Comestibles y Medicinales en Iberoamérica: investigación y Desarrollo en un entorno multicultural. México. ECOSUR INECOL. p. 29-38.

Guzmán, G. 2016. Las relaciones de los hongos sagrados con el hombre a través del tiempo. En Anales de Antropología 50 (1): 134-147. DOI: 10.1016/j.antro.2015.10.005.

Grupo Interdisciplinario para el Desarrollo de la Etnomicología en México (GIDEM). 2014. Grupo de Trabajo de la Sociedad Mexicana de Micología y de la Asociación Etnobiológica Mexicana A.C. Boletín No 8. Consultado 18 jun. 2017. Disponible en http://asociacionetnobiologica.org.mx/aem/ wp-content/uploads/Boletin-8-GIDEM-2.pdf

Jiménez-Ruiz A, H Thomé-Ortiz, C Burrola. 2016. Patrimonio biocultural, turismo micológico y etnoconocimiento. Revista el periplo sustentable. México, 29(30): 180-205. DOI: 10.21854/eps.v0i30.2529.

Knight D. 2014. Mushrooms, Knowledge Exchange and Polytemporality in Kalloni, Greek Macedonia. Food, culture society 17(2): 183-201. DOI: 10.2752/175174414X13871 910532105.

Lane B. 1994. What is rural tourism? Journal of Sustainable Tourism 2(1-2): 7-22. DOI: 10.1080/09669589409510680.

Lázaro A. 2008. El aprovechamiento micológico como vía de de- 
sarrollo rural en España: las facetas comercial y recreativa. Anales de Geografía 28(2): 111-136.

Le mycotourisme au Kamouraska: une initiative concertée Colloque sur les champignons forestiers et autres PFNL: Innovations et perspectives Le mercredi 27 août 2014 Pascale G. Malenfant Biopterre. Centre de développement des bioproduits. Consultado 19 jun. 2017. Disponible en http:// www.mycotourismekamouraska.com/pdf/PascaleGMalenfantCOLLOQUE.pdf

Lipovetsky G y J Serroy. 2015. La estetización del mundo. Vivir en la época del capitalismo estético. Barcelona. Anagrama. 416 p.

Mason M, A Paggiaro. 2009. Celebrating local products: The role of food events. Journal of Foodservice Business Research 12(4): 364-383. DOI: 10.1080/15378020903344323.

Martínez E, J Sánchez, R Torija, J Vega. 2010. Turismo micológico y desarrollo sostenible del medio rural en Soria. XII Coloquio de Geografía del Turismo, Ocio y Recreación. 17 p.

Martínez-Peña F, G Giner, Y Tejedores, MJ Campo, D Francés, Y Muñoz. 2003. Primeros resultados del estudio del aprovechamiento micológico a partir de encuestas en la zona MYAS (Soria): recolección, micoturismo y ordenación del recurso. Soria. Actas del I Congreso Nacional de Micología Forestal Aplicada. 9 p.

Martínez-Peña F, J Oria, A Ágreda. 2011. Manual para la gestión del recurso micológico forestal en Castilla y León. España. SOMACYL-Junta de Castilla y León. 451 p.

McLain RJ. 2008. Constructing a Wild Mushroom Panopticon: The Extension of Nation-State Control over the Forest Understory in Oregon, USA. Economic Botany 62(3): 343355. DOI. 10.1007/s12231-008-9025-8.

Micodata. Proyecto. 2013. Instituto Europeo de Micología. Consultado 23 mar. 2015. Disponible en http://www.micosylva. com/content/proyecto-micodata.

MICOSYLVA. 2013. Instituto Europeo de Micología. Proyecto MICOSILVA. Consultado 23 mar. 2015 Disponible en http://www.micosylva.com/content/proyecto-micosylva-1.

Montoya A, A Kong, R Garibay-Origel, C Méndez-Espinoza, R Tulloss, A Estrada-Torres. 2014. Availability of Wild Edible Fungi in La Malinche National Park, Mexico. Journal of Mycology 4: 1-15. DOI: 10.1155/2014/2418062014.

Montoya S, CE Orrego. 2012. Growth, fruiting and lignocellulolytic enzyme production by the edible mushroom Grifola frondosa (maitake). World Journal of Microbiology and Biotechnology 28(4): 1533-1541. DOI: 10.1007/s11274011-0957-2.

Moreno FA, R Garibay-Orijel, VJ Tovar, J Cifuentes. 2001. Situación actual de la etnomicología en México y el mundo. Etnobiología 1: 75-84.

Padilla ML. 2014. Diseño de una ruta micoturística en el municipio de Tequila, Jalisco, México, con base en un estudio etnomicológico. Tesis de Licenciatura en Biología. México. Universidad de Guadalajara. $61 \mathrm{p}$.

Pérez-Silva, E. y T Herrera. 2015. Exposiciones micológicas y congresos realizados por la Sociedad Mexicana de Micología. Revista Mexicana de Micología 42: 71-76.

Reyna S, S Garcia. 2014. Black truffle cultivation: a global reality. Forest Systems 23(2): 317-328. DOI: 10.5424/ fs/2014232-04771.

Ruán-Soto F, R Garibay-Orijel, J Cifuentes. 2004. Conocimiento micológico tradicional en la planicie costera del Golfo de México. Revista Mexicana de Micología 19: 57-70.

Ruán-Soto, F, J Cifuentes, R Mariaca, F Limón, L Pérez-Ramírez, S Sierra. 2009. Uso y manejo de hongos silvestres en dos comunidades de la selva lacandona, Chiapas, México. Revista Mexicana de Micología 29: 61-72.

Sabaté R, M Sogues, R Basora, LJ Romero. 2010. La valorización del patrimonio forestal como recurso ecoturístico en zonas de montaña: el caso del Mig Pallars y el Parque Natural del Alt Pirineu (Pirineo de Lérida, Cataluña). Ager. Revista de Estudios sobre Despoblación y Desarrollo Rural 9: 31-62.

Salafsky N, E Wollenberg. 2000. Linking livelihoods and conservation: A conceptual framework and scale for assessing the integration of human needs and biodiversity. World Development 28(8): 1421-1438. DOI: 10.1016/S0305750X(00)00031-0.

Scottish Fungi. 2014. Grampiam Fungus Group. Newsletter. Nó. 18. Consultado 16 jun. 2017. Disponible en https://sites. google.com/site/scottishfungi/local-groups/grampian-fungus-group/gfg-newsletter-archive/gfg-newsletter-2014

Secretaría del Convenio sobre la Diversidad Biológica. 2009. Gestión forestal sostenible, biodiversidad y medios de vida: Guía de buenas prácticas. Montreal, Canadá. IUC. 53 p.

Toledo VM, V Barrera, N Bassols. 2008. La memoria biocultural. La importancia ecológica de las sabidurías tradicionales. Barcelona, España. Icaria. 232 p.

Thomé-Ortiz H. 2008. Turismo rural y campesinado, una aproximación social desde la ecología, la cultura y la economía. Convergencia 15(47): 237-261.

Thomé-Ortiz H. 2015. Turismo micológico: una nueva mirada al bosque. Ciencia y Desarrollo 277: 14-19.

Thomé-Ortiz H. 2016. Turismo Rural y Sustentabilidad: El caso del turismo micológico en el estado de México. En Carreño F, A Vázquez coords. Ambiente y Patrimonio Cultural. México. CEDES, UAEMEX. p. 43-71.

Vantomme P. 2003. Setas silvestres comestibles de los bosques de la región pacífica noroccidental de América: un producto forestal no maderero rentable. UNASYLVA (FAO). 54(212): 46-47. ISSN 0251-1584.

Villaseñor IL, M Cedano, DL Guzmán. 2011. Propuesta sobre el desarrollo de una ruta micoturística en la sierra de Quila. Memorias I Foro de Conocimiento, uso y gestión del Área Natural Protegida Sierra de Quila. p. 87-89.

Zambonelli A, M Iotti, I Hall. 2015. Current status of truffle cultivation: recent results and future perspectives. Micologia Italiana. 44: 31-40. DOI: 10.6092/issn.2465-311X/5593.

Zizumbo-Villarreal L, C Burrola-Aguilar y M Hernández. 2012. El Micoturismo como alternativa de desarrollo local en Amanalco de Becerra, México. In Cristovão A, X Pereiro eds. Atas do VIII CITURDES - Congresso Internacional de Turismo Rural e Desenvolvimento Sustentável: Turismo Rural em Tempos de Novas Ruralidades. UTAD, CETRAD. p. 825-839. 